\section{Estudo \\ CoDebate}

em Cestão

Plamejamento
Revista Estudo \& Debate, Lajeado, v. 24, n. 2, 2017. ISSN 1983-036X

DOI: http://dx.doi.org/10.22410/issn.1983-036X.v24i2a2017.1243

\title{
TRANSPARENCIA, ACCOUNTABILITY Y GOBIERNO ABIERTO: COMPARACIÓN DE LOS MUNICIPIOS DE HERMOSILLO, SONORA Y JUÁREZ, CHIHUAHUA DESDE LA ACREDITACIÓN CIUDADANA DE LA FUNCIÓN CONTRALORA DE LOS MUNICIPIOS EN MÉXICO' ${ }^{1}$
}

\author{
Abraham Paniagua Vázquez², Miguel Arturo Morales Zamorano ${ }^{3}$
}

\begin{abstract}
Resumen: Considerando como base las propuestas teórico-conceptuales de la transparencia, la accountability (rendición de cuentas) y el gobierno abierto, este trabajo mantiene como objetivo discutir sobre los resultados de la "Matriz de diagnóstico de la acreditación ciudadana de la función contralora" en los municipios de Hermosillo, Sonora y Juárez, Chihuahua, una herramienta que busca la gestión evaluadora ciudadana a sistemas administrativos públicos con la finalidad de acotar la discrecionalidad y disminuir la tendencia histórica de opacidad y ocultamiento mediante la colaboración socio-gubernamental. Se percibe a este ejercicio como continuidad de los esfuerzos ciudadanos por involucrarse a las dinámicas internas de los distintos órganos públicos, en este caso dos municipios del norte de México que comparten ciertas características y mantienen diferencias que en conjunto, los posicionan como atractivos para su análisis. Se concluye en general, que prácticas como ésta son necesarias para la consolidación de la vida democrática mexicana y por ende para el gobierno abierto, ya que se implementan estrategias "desde abajo" que superan visiones estructurales básicas consideradas en la evaluación de la cosa pública, como los procesos electorales.
\end{abstract}

Palabras clave: Gobierno abierto. Transparencia. Accountability. Hermosillo. Juárez.

1 Esta investigación es producto del proyecto de investigación "Acreditación ciudadana de la función contralora de los municipios en México" en el que participan los cuerpos académicos CA-UACH-120 "Política, Gobierno y Sociedad" de la Universidad Autónoma de Chihuahua, y el CA-USON-175 "Desarrollo humano, económico, comunitario e institucional" adscrito a la Universidad de Sonora.

2 Doctor en Ciencias Sociales, especialidad en Política, Gobierno y Asuntos Públicos, adscrito a la Universidad Autónoma de Chihuahua, Facultad de Ciencias Políticas y Sociales. Miembro del Sistema Nacional de Investigadores del Consejo Nacional de Ciencia y Tecnología Nivel I Recibe comentarios en abrahampaniagua@hotmail.com

3 Doctor en Ciencias, especialidad en Ciencias Administrativas, adscrito a la Universidad de Sonora, Departamento de Sociología y Administración Pública, Miembro del Sistema Nacional de Investigadores del Consejo Nacional de Ciencia y Tecnología Nivel I. Recibe comentarios en moralesz@sociales.uson.mx 


\title{
TRANSPARENCY, ACCOUNTABILITY AND OPEN GOVERNMENT: COMPARISON OF THE MUNICIPALITIES OF HERMOSILLO, SONORA, AND JUAREZ, CHIHUAHUA FROM PUBLIC ACCREDITATION OF THE COMPTROLLER FUNCTION OF MUNICIPALITIES IN MEXICO
}

\begin{abstract}
Summary: Considering proposals as a basis the theoretical-conceptual of the transparency, accountability (accountability) and open government, this work maintains as objective to discuss on the results of the "diagnostic matrix of the accreditation of the citizen comptroller function" in the municipalities of Hermosillo, Sonora and Juarez, Chihuahua, a tool that searches for the citizen management evaluation to public administrative systems with the purpose of limiting the discretion and decrease the historical trend of opacity and concealment through collaboration partner-government. It is perceived to this exercise as a continuity of the efforts to engage citizens to the internal dynamics of the various public bodies, in this case, two municipalities in the north of Mexico that share certain characteristics and differences that remain on the whole, the positioned themselves as attractive for their analysis. It is concluded in general, that practices such as this are necessary for the consolidation of democratic life mexican and hence for open government, already being implemented strategies "from below" visions that exceed basic structural considered in the evaluation of the public thing, such as the electoral process.
\end{abstract}

Keywords: Open Government. Transparency. Accountability. Hermosillo. Juárez.

\section{Presentación}

La incorporación del gobierno abierto al léxico de la política ha dado pie a varias vertientes de investigación como: la colaboración ciudadana en asuntos gubernamentales, su participación en gobernanza en red, el acceso público a la información y las presiones globales para generar las condiciones sobre la transparencia y el acceso a la información pública.

Este trabajo es parte de esas tendencias, sólo que como resultado de estudios de ciudadanos en dos Estados vecinos y fronterizos ambos con Estados Unidos (Sonora y Chihuahua), estudios de académicos que pueden ir dando pauta para incentivar la práctica del gobierno abierto, en franca oposición a tradicionales formas culturales de ocultar información (Epstein,1996), en este caso sobre procesos que se desarrollan al interior de las administraciones públicas de dos municipios, uno de cada uno de los Estados, de Hermosillo en el Estado de Sonora y Juárez en el Estado de Chihuahua.

La percepción ciudadana de la existencia de la corrupción como fallo endémico es confirmada por Transparencia Internacional (TI, 2016), al precisar que México se ubicó en el último lugar entre los países que integran la Organización para la Cooperación y el Desarrollo Económico (OCDE) y al menos 40 posiciones por debajo de sus principales competidores económicos como es el caso de China, India y Brasil, lo cual invita a plantearse preguntas de investigación en torno al rol y responsabilidades ciudadanas y entre otras, de autoridades municipales para abatir este $\mathrm{flagelo}^{4}$, siendo estas últimas quienes podrían llegar

4 El 2014 Corruption Perception Index de Transparency International sitúa a México en el lugar 103 de los 175 países evaluados, mientras que para 2016 lo sitúa en el lugar 123. 
a promover e impulsar mecanismos para generar confianza ciudadana, dada su cercanía y posibilidades de articulación conjunta.

El propósito de este paper es compartir los hallazgos derivados de la aplicación de la "Matriz de diagnóstico para la acreditación ciudadana de la función contralora", una herramienta que incentiva la colaboración social con relación a la tendencia del gobierno abierto en los municipios de Hermosillo, Sonora y de Juárez, Chihuahua, específicamente las administraciones 2012 - 2015 y 2010 - 2013 de manera respectiva.

Ambos municipios norteños comparten similitudes interesantes: son los más poblados de sus estados 5 , han consolidado períodos de gobierno local de transición que reafirman la existencia de una cultura política basada en el bipartidismo (Revolucionario Institucional Acción Nacional), ${ }^{6}$ y, se posicionan como dos de los más endeudados de México. ${ }^{7}$

Para efectos de influencia hacia el incentivo del Gobierno Abierto, un matiz para subrayar es la condición hermosillense como capital estatal, trascendente, ya que es la sede de los poderes ejecutivo, legislativo y judicial, así como de las dependencias públicas federales y estatales y de instancias como el instituto local para la transparencia y el acceso a la información pública. Juárez no cuenta con esos elementos.

La idea principal que guía este trabajo es que la colaboración, como participación social corresponsable y organizada configura la política del gobierno abierto. Es decir, la ciudadanía se erige como actor activo, ya que genera las capacidades necesarias no sólo para solicitar información, sino que además evalúa el papel de los gobiernos, en este caso dos municipales.

La estrategia de recolección de información contempló dos momentos: el primero enfocado a una revisión in situ de documentos y evidencias que respaldaran las respuestas a las áreas e indicadores incluidos en la matriz bajo comento; un segundo momento consistió en un proceso de concertación con funcionarios de las dependencias: Contraloría, Tesorería, Secretaría Técnica y Coordinación General de Planeación y Evaluación de la administración municipal de Juárez 2010 - 2013; mientras que personal de la Contraloría Municipal de Hermosillo administración 2012 - 2015 fueron los encargados de responder a la misma dinámica. El criterio de selección de estas instancias responde a que son las señaladas en

5 Hermosillo cuenta con 784,342 habitantes que representan el 29.46\% de la población en el estado de Sonora, por su parte Juárez tiene una población de 1,332,131 habitantes, el 39.11\% de los chihuahuenses (Inegi, 2010).

6 El primer presidente municipal hermosillense emanado de un partido distinto al Nacional Revolucionario, de la Revolución Mexicana o Revolucionario Institucional se eligió en 1967 y perteneció a Acción Nacional, a partir de ese año han sido recurrentes las transiciones Pri-Pan en la alcaldía de Hermosillo. Por su parte, el primer gobierno municipal no priista en Juárez se eligió en 1983 y perteneció a Acción Nacional, esta elección marcó un interesante período de intercambio Pri-Pan en la presidencia municipal.

7 La Secretaría de Hacienda y Crédito Público Shcp detecta una deuda hermosillense de 1,401.3 millones de pesos, la quinta mayor en el país. Mientras que el municipio juarense contrató un crédito por 2,200 millones de pesos para financiar el Plan de Movilidad Urbana, una estrategia de mejoramiento, construcción y reparación de algunas de las avenidas más transitadas, esta deuda se hará oficial una vez concluyan todas las obras contempladas. 
los manuales de operación vigentes como las responsables de gestionar y/o controlar los recursos municipales.

Los apartados de este artículo son cuatro: en el primero se presentan las aproximaciones conceptuales a la transparencia, la accountability y el gobierno abierto; a manera de señalar el contexto de este ejercicio en el segundo apartado se exponen y analizan cuatro índices sobre aspectos relacionados al funcionamiento de los municipios más importantes de los estados de la frontera norte de México; durante el tercero se analiza la información recabada ex profeso mediante la Matriz de diagnóstico de la acreditación ciudadana de la función contralora así como también se emiten las recomendaciones producto del mismo análisis; el cuarto incluye las conclusiones.

\section{Transparencia, accountability y gobierno abierto: Una aproximación conceptual}

Para efectos de dimensionar la relevancia del presente artículo, habremos de referirnos a que la transparencia y la rendición de cuentas ofrecen su sentido de necesidad en la medida de que sancionan e inhiben la comisión de actos ilícitos y son medida de la conducta ética, siendo también útiles como medida de regulación social de las decisiones que se toman a nombre de todos (FERREIRO1999; MERINO,2005), presentándose a la luz del primer autor, las modalidades activa y pasiva de transparencia, esto es, cuando se difunde por obligación legal, de manera periódica y sistematizada la información pública relacionada a sus actividades para que la sociedad evalúe, mientras que la transparencia pasiva obliga en su caso a facilitar a los ciudadanos que lo soliciten el acceso a información pública que obre en poder de dependencias gubernamentales ${ }^{8}$. Sin transparencia activa, a manera de rendir cuentas sobre las actividades que realizan instituciones gubernamentales, el ciudadano recurre entonces a la transparencia pasiva, y sólo disponiendo de este derecho es posible cierta coadyuvancia o trabajo colaborativo conjunto que permita arribar a condiciones de mejoría en credibilidad, satisfacción y confianza ciudadana.

Sobre esto último, hemos de estar de acuerdo, que cualquier teórico de la democracia y nosotros mismos hemos de reafirmar que tratándose de asuntos públicos, las acciones de quienes administran recursos para ello han de sujetarse a escrutinio público, permitiendo además, de ser posible, a los ciudadanos, que se constituyan mínimamente en vigilantes del desempeño burocrático, a fin de

[...] dotar de legitimidad y credibilidad al régimen político, mientras que la ciudadanía va adquiriendo madurez política e interés por la correcta marcha de su gobierno, evitando la cultura de la opacidad, propia de sociedades en las que se niega la presencia de ciudadanos libres (Rubia, Martínez y Parra, 2011; Uvalle, 2008; Emmerich, 2004; O’Donnell, 1998; Prud'homme, 1997). (PANIAGUA; MORALES, 2015)

8 Es de hacer notar a este respecto que hay tres tipos de información: la pública, la personal o privada y la reservada, siendo sólo la primera la cual debe hacerse accesible a la ciudadanía por ley, mientras que la información personal o privada se protege, así como la que el Estado haya determinado para efectos de seguridad nacional o Estatal como reservada. 
Asimismo, y en congruencia con lo anterior, tiende a percibirse cierta tendencia a que la ciudadanía tome parte activa, directa y efectiva en la vigilancia y la conducción de los asuntos públicos (EMMERICH, 2004), ello en la idea de actualidad de combatir la corrupción, cuyas implicaciones impactan negativamente en la credibilidad de las instituciones, en el desarrollo económico de la sociedad y en general, sobre las condiciones de vida de la población (REYES, 2004). También la transparencia y la rendición de cuentas generan la ventaja institucional de conocer cuándo, dónde, por qué y cómo las autoridades actúan para dar vida a los procesos de gobierno (UVALLE, 2008), siendo condición necesaria para la sobrevivencia de la democracia, ya que permite el acceso ciudadano oportuno a la información relativa al desempeño gubernamental (LÓPEZ, 2005).

Sin embargo, 'Los representados no creen que los representantes actúen en su nombre o en el del bien común” (MAINWARNING; BEJARANO; LEÓN-GÓMEZ, 2008, p. 45-46), lo cual deja de representar incentivo para colaborar e invertir parte de su tiempo, esfuerzo y hasta dinero en la idea de mejorar gradualmente las disfunciones detectadas de instituciones de gobierno, presentándose este fenómeno como todo un obstáculo al ejercicio social por influir en lo público como parte de la crisis de la representación democrática. ${ }^{9}$ Esta crisis revela el componente subjetivo de comportamiento mediante el cual se refleja la insatisfacción ciudadana con la manera en la que son representados, o en su caso el sentimiento de nula representación. Por ello la apuesta de este estudio, de fomentar la colaboración inicialmente en la medición de la gestión municipal a partir del cambio en la cultura de lo público y el compromiso por la colaboración (NASER; CONCHA; PARRA, 2012), lo cual impulsa, además, la necesidad de gobiernos abiertos.

Los gobiernos abiertos, estaremos de acuerdo en esto, son teórica y pragmáticamente toda una estrategia para superar los problemas señalados de la democracia representativa y acercarse al modelo de democracia deliberativa y resolutiva a partir de la colaboración, de la práctica de valores, de repensar administraciones y gobiernos, sus procedimientos y normas (CALDERÓN, 2012). De hecho, la función relacional de este espectro teórico ya en la práctica exige per se, un cierto liderazgo de organizaciones corporativas generalmente fuera, pero de manera paralela, del poder gubernamental (MARIŃEZ NAVARRO, 2016). "El concepto mismo apuesta por la aplicación de acciones innovadoras y efectivas que, bajo un funcionamiento óptimo en su diseño e implementación, podrían servir de vehículo para una transformación genuina del propio gobierno" (WEERAKKODY; REDDICK, 2013 , p. 8-9). Se apuesta por un diseño o rediseño institucional para gobiernos municipales abiertos (HERNÁNDEZ, 2016), y que sea consistente con las aspiraciones de legitimidad y credibilidad ciudadana. Así, su lógica se fundamenta en tres elementos: transparencia en la acción, procesos y datos del gobierno; participación al interior del gobierno con las personas, lo cual permite generar nuevas ideas para estimular el desempeño del personal (ARTHUR; KOLSON, 2017) y con ello resolver problemas sociales; y colaboración para "[...] implicar de forma activa y real a los ciudadanos en la formulación y ejecución de políticas” (HERNÁNDEZ, 2012, p. 50).

9 La crisis de la representación es un concepto académico de intenso debate, sobre todo en democracias emergentes, algunas son producto de la "tercera ola" (HUNGTINGTON, 1996). 
El juego dialéctico entre transparencia - accountability en el marco de un gobierno abierto genera un lugar común: el espectro multicéntrico de actores interesados en acceder al poder de la toma de decisiones de los asuntos públicos, escenario reflejado en "la cabida de nuevos actores y movimientos que reflejan la pluralidad del espacio público" (UVALLE, 2008, p. 100). En este ejercicio de empoderamiento social emergen como actores de la política organizaciones de la sociedad civil, colectivos, movimientos urbanos y líderes sociales que hasta antes de esta apertura democrática, es decir durante la democracia representativa, sólo influían de manera indirecta y esporádica, especialmente durante el proceso electoral (PETERS; MURILLO, 2005).

El gobierno abierto demanda de la concertación de grupos de ciudadanos organizados y expertos en diversas ramas del quehacer institucional para exaltar el empoderamiento social (POLLO, 2012), como tal, es una estrategia a futuro condicionada por la puesta en marcha y correcta ejecución de múltiples proyectos en torno al concepto de participación ciudadana en propósitos públicos con intenciones de acrecentar su capacidad de influencia en ítems como la óptima gestión de los dineros, la incidencia en la agenda legislativa, proponer y evaluar políticas públicas o influir en la mejora continua de la efectividad de las diversas dependencias e instituciones que configuran a la administración pública.

Morales y Castro (2015, p. 13), ańaden a este breve recorrido en torno a la necesaria emancipación y empoderamiento ciudadano a través mucho más que de la simple participación, de la colaboración e integración a acciones gubernativas, que:

Un "Estado abierto", similar al open government se puede llegar a definir como un complejo de normas, cuya hechura ciudadana y multidisciplinaria, regula conductas de gobiernos y ciudadanos, apoyados en asociaciones civiles de expertos, financiadas y reconocidas por la misma ciudadanía dada su transparencia, colaboración y rendición de cuentas.

Siendo que esas asociaciones civiles de ciudadanos expertos incorporados a la acción gubernativa tienden a conceder efectividad, satisfacción, credibilidad, confianza, re-funcionalidad, legitimidad y fortaleza a sus instituciones gubernamentales, de manera colaborativa y especializada, desde fuera de las estructuras administrativas de gobiernos; a ese espectro de actuación ciudadana en Estados abiertos se puede llegar a denominar Gestión Socio-gubernamental.

Para lo anterior, se hace pertinente la apertura a la acción de ciudadanos expertos en Gestión de asuntos de gobierno y de administración de "lo público" que como ciudadanos actúen desde fuera de las instituciones de gobierno, fieles a ciudadanos y no a políticos en turno ni de partido, organizados en OSC (ONG's) en una Red multinacional capaz de presionar a pequeños gobiernos locales, sub-nacionales y nacionales a fin de que las políticas sean ahora sí públicas, siendo que éste tipo de Gestión desde los ciudadanos organizados, socialmente empoderados, tiende a perfilarse como "Gestión Socio-gubernamental", como marco teórico práctico tendencial de las políticas públicas (MORALES; BANDA, 2014, p. $11)$.

El reto entonces para el estímulo de la transparencia y rendición de cuentas es doble: por un lado los gobiernos deben generar en los ciudadanos condiciones para la colaboración activa generalizada y propiciar el sentido de corresponsabilidad en los asuntos de interés 
público; por el otro, la sociedad se ve involucrada en la coyuntura de generar estrategias ciudadanas y tácticas colectivas inteligentes que permitan inmiscuirse en tareas de interés general, en plena acción de la accountability societal (O’DONNEL, 2003).

Para los gobiernos sub-nacionales latinoamericanos en general, la consideración de patrones relacionales entre gobiernos y ciudadanía, a fin de lograr sinergias y efectos de mayor satisfacción e impacto del trabajo estatal se ha convertido en un pendiente (HEVIA, 2015).

\section{La participación ciudadana en la evaluación de los Municipios del norte de México: antecedentes para el ejercicio de acreditación}

Una de las matices auténticas para apostar por el gobierno abierto es que actúa como mecanismo generador de procedimientos y condiciones necesarios a la transparencia y rendición de cuentas, a través de los cuáles la sociedad requisa, se entera y evalúa las condiciones de los asuntos de interés general.

Solicitar información desde la sociedad, permitida para estimar administraciones gubernamentales, políticas públicas o cualquier acción de gobierno y comparar sus resultados, es una práctica que se ha vuelto recurrente en México. Hemos sido testigos de acciones organizadas dirigidas a discutir sobre el gasto del presupuesto público, a cerca de los avances en las tendencias del gobierno electrónico o relativas al cumplimiento o incumplimiento de las responsabilidades de la burocracia y representantes populares. En su conjunto, exhiben a sociedades en vías de consolidación democrática que generan alternativas de participación ciudadana a las tradicionales, como emitir el sufragio.

Los municipios del norte de México como lo son Hermosillo en el Estado de Sonora y Juárez en el Estado de Chihuahua, han sido objeto de varias y distintas evaluaciones desde la sociedad organizada, de utilidad para comparar características de los dos municipios bajo requisa. El Índice de Competitividad Urbana 2014 construido por el Instituto Mexicano para la Competitividad ImCO evalúa 78 zonas metropolitanas a través de 10 subíndices que contienen 90 indicadores. mide la capacidad de las ciudades mexicanas para atraer y retener talento e inversiones.

Los resultados de este ejercicio (CUADRO 1) colocan a la ciudad de Hermosillo como la décima nacional en cuanto a competitividad urbana y la cuarta entre los estados de la frontera norte, resaltan los subíndices de Sociedad incluyente, preparada y sana y Sectores precursores. Los pendientes importantes abarcan el Manejo sustentable del medio ambiente y el Sistema político estable y funcional. 
Cuadro 1 - Índice de Competitividad Urbana 2014. Resultados de las principales ciudades del norte de México (estados fronterizos)

\begin{tabular}{|l|l|c|c|c|c|c|c|c|c|c|c|c|}
\hline Ciudad & $\begin{array}{c}\text { Nivel de } \\
\text { Competitividad }\end{array}$ & $\begin{array}{c}\text { Lugar } \\
\text { General }\end{array}$ & i & ii & iii & iv & $\mathbf{v}$ & vi & vii & viii & ix & $\mathbf{x}$ \\
\hline Monterrey & Alta & 4 & 17 & 37 & 8 & 78 & 14 & 6 & 8 & 5 & 6 & 3 \\
\hline Saltillo & Adecuada & 7 & 12 & 51 & 32 & 11 & 16 & 1 & 34 & 19 & 27 & 6 \\
\hline Chihuahua & Adecuada & 9 & 64 & 13 & 9 & 54 & 10 & 13 & 40 & 6 & 7 & 12 \\
\hline Hermosillo & Adecuada & 10 & 14 & 75 & 2 & 52 & 11 & 5 & 21 & 4 & 22 & 8 \\
\hline Monclova & Media Alta & 21 & 20 & 52 & 44 & 10 & 16 & 2 & 75 & 27 & 40 & 11 \\
\hline Tampico & Media Alta & 22 & 22 & 35 & 37 & 25 & 47 & 17 & 37 & 15 & 17 & 48 \\
\hline $\begin{array}{l}\text { Ciudad } \\
\text { Victoria }\end{array}$ & Media Alta & 24 & 6 & 7 & 3 & 43 & 45 & 22 & 61 & 11 & 60 & 55 \\
\hline $\begin{array}{l}\text { Ciudad } \\
\text { Obregón }\end{array}$ & Media Alta & 28 & 39 & 46 & 6 & 55 & 17 & 42 & 56 & 12 & 49 & 21 \\
\hline Mexicali & Media Alta & 33 & 76 & 12 & 47 & 59 & 24 & 41 & 18 & 30 & 18 & 33 \\
\hline Piedras Negras & Media Alta & 34 & 19 & 24 & 60 & 15 & 40 & 64 & 77 & 23 & 30 & 10 \\
\hline Tijuana & Media Alta & 37 & 62 & 14 & 48 & 68 & 18 & 33 & 59 & 16 & 29 & 54 \\
\hline Ciudad Juárez & Media Baja & 43 & 63 & 1 & 31 & 73 & 53 & 11 & 62 & 44 & 21 & 60 \\
\hline La Laguna & Media Baja & 48 & 73 & 57 & 59 & 50 & 49 & 3 & 42 & 38 & 26 & 27 \\
\hline Reynosa & Media Baja & 54 & 8 & 19 & 67 & 71 & 62 & 35 & 71 & 49 & 38 & 63 \\
\hline Matamoros & Media Baja & 59 & 10 & 22 & 63 & 76 & 56 & 15 & 76 & 21 & 45 & 70 \\
\hline Ensenada & Media Baja & 65 & 71 & 58 & 50 & 60 & 48 & 66 & 29 & 57 & 51 & 51 \\
\hline Nuevo Laredo & Baja & 74 & 74 & 28 & 61 & 77 & 73 & 47 & 69 & 62 & 46 & 46 \\
\hline
\end{tabular}

Fuente: Elaboración propia con base a Imco (2014). i. Sistema de derecho confiable y objetivo. ii. Manejo sustentable del medio ambiente. iii. Sociedad incluyente, preparada y sana. iv. Sistema político estable y funcional. v. Gobiernos eficientes y eficaces. vi. Mercado laboral. vii. Economía estable. viii. Sectores precursores. ix. Aprovechamiento de las Relaciones Internacionales. x. Innovación en los sectores económicos.

Por su parte, Ciudad Juárez ocupa el lugar 43 general en competitividad urbana y el 12 en relación a las ciudades de los estados de la frontera norte. Los mejores subíndices evaluados fueron (CUADRO 1) el Manejo sustentable del medio ambiente y su Mercado laboral, se detectaron problemas en el Sistema político estable y funcional y en el Sistema de derecho confiable y objetivo.

El Índice de herramientas electrónicas de los Gobiernos Locales, también del IMcO contempló la revisión de 54 municipios mediante 4 secciones que contienen 48 indicadores.

La ciudad de Hermosillo resultó la mejor evaluada (CUADRO 2) entre las más importantes de los estados fronterizos y la tercera a nivel nacional. La sección que resalta es Transacción (primer lugar nacional), que evalúa la oferta hacia la ciudadanía para realizar procedimientos o trámites en línea. En el lado opuesto se encuentra la sección Experiencia 
del usuario (lugar 11 nacional), califica si el diseño del portal permite al usuario encontrar la información que necesita.

Cuadro 2 - Índice de herramientas electrónicas de Gobiernos Locales. Resultados de los principales municipios del norte de México (estados fronterizos)

\begin{tabular}{|l|c|c|c|c|c|}
\hline \multicolumn{1}{|c|}{ Municipio } & $\begin{array}{c}\text { Lugar } \\
\text { general/ } \\
\text { puntaje }\end{array}$ & $\begin{array}{c}\text { i } \\
\text { Información } \\
\text { disponible }\end{array}$ & $\begin{array}{c}\text { ii } \\
\text { Interacción }\end{array}$ & $\begin{array}{c}\text { iii } \\
\text { Transacción }\end{array}$ & $\begin{array}{c}\text { iv } \\
\text { Experiencia } \\
\text { del usuario }\end{array}$ \\
\hline Hermosillo & $3 / 73.2$ & $9 / 87.50$ & $2 / 61.54$ & $1 / 93.75$ & $11 / 50.00$ \\
\hline Chihuahua & $13 / 53.0$ & $27 / 68.75$ & $14 / 42.31$ & $22 / 37.50$ & $4 / 63.64$ \\
\hline Tijuana & $16 / 48.7$ & $12 / 84.38$ & $18 / 34.62$ & $7 / 56.25$ & $46 / 22.73$ \\
\hline Guadalupe & $22 / 41.8$ & $16 / 78.13$ & $31 / 23.08$ & $30 / 25.00$ & $20 / 40.91$ \\
\hline Monterrey & $24 / 40.4$ & $12 / 84.38$ & $30 / 25.08$ & $27 / 31.25$ & $46 / 22.73$ \\
\hline Torreón & $32 / 35.8$ & $18 / 75.00$ & $36 / 19.23$ & $40 / 12.50$ & $23 / 36.36$ \\
\hline Ciudad Juárez & $35 / 35.4$ & $15 / 78.13$ & $36 / 19.23$ & $40 / 12.50$ & $34 / 31.82$ \\
\hline $\begin{array}{l}\text { San Nicolás de los } \\
\text { Garza }\end{array}$ & $37 / 34.6$ & $23 / 71.88$ & $46 / 11.54$ & $34 / 18.75$ & $23 / 36.36$ \\
\hline Mexicali & $38 / 34.4$ & $39 / 59.38$ & $31 / 23.08$ & $34 / 18.75$ & $23 / 36.36$ \\
\hline Saltillo & $46 / 30.8$ & $42 / 56.25$ & $36 / 19.23$ & $30 / 25.00$ & $46 / 22.73$ \\
\hline Reynosa & $51 / 24.9$ & $52 / 37.50$ & $25 / 26.92$ & $40 / 12.50$ & $46 / 22.73$ \\
\hline Matamoros & $54 / 14.6$ & $54 / 28.13$ & $50 / 7.69$ & $54 / 0.00$ & $46 / 22.73$ \\
\hline
\end{tabular}

Fuente: Elaboración propia con base a Imco (2015).

Los resultados de este mismo índice (CUADRO 2), sitúan a Ciudad Juárez en el escalafón 35 nacional y 7 regional. La mejor sección es Información disponible (lugar 15 nacional), que mide la cantidad y calidad de información que los municipios ponen a disposición de los usuarios. Mientras que la sección peor evaluada es Transacción (lugar 45 nacional), descrita líneas arriba.

El IMCO también propone el Índice de información municipal presupuestal 2014 que verifica el cumplimiento de las obligaciones de contabilidad gubernamental de 394 municipios y 16 delegaciones con base a 9 secciones (i. Acceso inicial, ii. Aspectos generales, iii. Clasificaciones, iv. Poderes, dependencias, organismos, v. Tabuladores-plazas, vi. Deuda pública, vii. Recursos federales, viii. Rubros específicos, ix. Criterios) y 80 criterios.

Lo que respecta a este escrutinio (CUADRO 3) proyecta al Municipio de Juárez como el octavo con relación a los más importantes de la zona norte, ya que cumplió con el $42.50 \%$ de los criterios solicitados (34 de 80), por su parte el Municipio de Hermosillo se ubica en la posición 13 con el 40\% de cumplimiento (32 de 80). 
Cuadro 3 - Índice de información municipal presupuestal 2014. Resultados de los principales municipios del norte de México (estados fronterizos)

\begin{tabular}{|c|c|c|c|c|c|c|}
\hline \multirow{2}{*}{ Municipio } & \multirow{2}{*}{ Presupuesto } & \multirow{2}{*}{ Habitantes* } & \multirow{2}{*}{$\begin{array}{c}\text { Presupuesto } \\
\text { por } \\
\text { habitante } \\
\end{array}$} & \multicolumn{3}{|c|}{ Evaluación } \\
\hline & & & & Aciertos & $\%$ & Lugar \\
\hline Saltillo & $\$ 1,908,836,000.00$ & 725,123 & $\$ 2,632.43$ & 79 & $98.75 \%$ & 1 \\
\hline Torreón & $\$ 2,112,640,717.72$ & 639,629 & $\$ 3,302.92$ & 74 & $92.50 \%$ & 2 \\
\hline Monclova & $\$ 428,937,362.61$ & 216,206 & $\$ 1,983.93$ & 70 & $87.50 \%$ & 3 \\
\hline Nuevo Laredo & $\$ 2,466,771,803.94$ & 384,033 & $\$ 6,423.33$ & 38 & $47.50 \%$ & 4 \\
\hline Chihuahua & $\$ 2,327,938,005.18$ & 819,543 & $\$ 2,840.53$ & 37 & $46.25 \%$ & 5 \\
\hline Reynosa & $\$ 1,401,705,595.64$ & 608,891 & $\$ 2,302.06$ & 35 & $43.75 \%$ & 6 \\
\hline Victoria & $\$ 771,516,000.00$ & 321,953 & $\$ 2,396.36$ & 35 & $43.75 \%$ & 6 \\
\hline Juárez & $\$ 3,291,011,261.97$ & $1,332,131$ & $\$ 2,470.49$ & 34 & $42.50 \%$ & 8 \\
\hline $\begin{array}{l}\text { San Pedro Garza } \\
\text { García }\end{array}$ & $\$ 1,442,601,090.00$ & 122,659 & $\$ 11,761.07$ & 33 & $41.25 \%$ & 9 \\
\hline Monterrey & $\$ 3,921,408,290.55$ & $1,135,550$ & $\$ 3,453.31$ & 33 & $41.25 \%$ & 9 \\
\hline Cajeme & $\$ 1,077,983,678.46$ & 409,310 & $\$ 2,633.66$ & 33 & $41.25 \%$ & 9 \\
\hline Matamoros & $\$ 1,100,013,170.00$ & 489,193 & $\$ 2,248.63$ & 33 & $41.25 \%$ & 9 \\
\hline Hermosillo & $\$ 2,419,887,768.58$ & 784,342 & $\$ 3,085.25$ & 32 & $40 \%$ & 13 \\
\hline Mexicali & $\$ 3,054,875,349.99$ & 936,826 & $\$ 3,260.88$ & 26 & $32.50 \%$ & 14 \\
\hline Ensenada & $\$ 1,418,352,867.00$ & 466,814 & $\$ 3,038.37$ & 22 & $27.50 \%$ & 15 \\
\hline Guadalupe & $\$ 1,463,849,881.00$ & 678,006 & $\$ 2,159.05$ & 19 & $23.75 \%$ & 16 \\
\hline Tijuana & $\$ 4,919,600,185.98$ & $1,559,683$ & $\$ 3,154.23$ & 3 & $3.75 \%$ & 17 \\
\hline
\end{tabular}

Fuente: Elaboración propia con base a Imco (2014b), *INEGI (2010).

A la par de los resultados generados (CUADRO 3), el Índice de información municipal presupuestal expone matices interesantes, por ejemplo: Hermosillo, que cuenta con menor población que Juárez $(783,342$ por $1,332,131$ habitantes) recibe mayor presupuesto por habitante $(\$ 3,085.25$ por $\$ 2,470.49)$, de hecho, el Municipio de Juárez invierte menor cantidad de recursos por habitante $(\$ 2,470.49)$ que Tijuana $(\$ 3,154.23)$ y Monterrey $(\$ 3,453.31)$ los tres municipios con más de un millón de habitantes en la región. De las capitales de los estados de la frontera norte mexicana, la que más invierte por habitante es Mexicali $(\$ 3,260.88)$, seguida de Hermosillo $(\$ 3,085.25)$. Desafortunadamente mayor inversión pública no se relaciona con mayor eficiencia en la gestión de información municipal presupuestal, ya que San Pedro Garza García en Nuevo León es el que más invierte por habitante $(\$ 11,761.07)$ y cumple sólo con el $41.25 \%$ de los criterios bajo análisis (33 de 80). 
Por su parte, la consultora Gabinete de Comunicación Estratégica GcE diseñó el Índice de calidad de vida 2014, implica la evaluación ciudadana de 10 variables. Hermosillo se ubica en el lugar nacional 19 y 8 regional con una puntuación de 73.4. Ciudad Juárez alcanzó el lugar nacional 69 y 11 en la región (CUADRO 4).

Cuadro 4 - Índice de calidad de vida 2014. Resultados de los principales municipios del norte de México (estados fronterizos)

\begin{tabular}{|l|c|c|c|c|c|c|c|c|c|c|}
\hline \multicolumn{1}{|c|}{ Ciudad } & $\begin{array}{c}\text { Puntuación } \\
\text { Total }\end{array}$ & $\begin{array}{c}\text { Lugar } \\
\text { Nacional }\end{array}$ & i & ii & iii & iv & v & vi & vii & viii \\
\hline Monterrey & 80 & 5 & 2.6 & 2.5 & 2.4 & 3.3 & 3 & 3 & 2.9 & 2.9 \\
\hline Saltillo & 79.3 & 7 & 3 & 2.9 & 2.3 & 2.9 & 2.9 & 3.2 & 2.8 & 2.9 \\
\hline Chihuahua & 77.8 & 8 & 2.6 & 2.8 & 2.4 & 3 & 2.9 & 3 & 2.9 & 2.9 \\
\hline Nuevo Laredo & 77.8 & 8 & 3 & 3 & 2.4 & 2.9 & 3 & 2.7 & 2.4 & 2.7 \\
\hline Ciudad Obregón & 76.9 & 12 & 2.8 & 2.7 & 2.3 & 2.9 & 2.9 & 2.6 & 3.1 & 3 \\
\hline Matamoros & 74 & 18 & 2.8 & 2.8 & 2.3 & 2.8 & 2.9 & 2.5 & 2.5 & 2.7 \\
\hline Hermosillo & 73.4 & 19 & 2.6 & 2.6 & 2.4 & 3 & 3 & 2.4 & 2.3 & 2.8 \\
\hline Ciudad Victoria & 73.4 & 219 & 2.8 & 2.9 & 2.3 & 2.7 & 2.9 & 2.5 & 2.7 & 2.7 \\
\hline Torreón & 70.7 & 30 & 2.8 & 2.4 & 2.3 & 2.8 & 2.9 & 2.7 & 2.3 & 2.6 \\
\hline Mexicali & 69.9 & 33 & 2.8 & 2 & 2.2 & 3 & 2.9 & 2.4 & 2.4 & 2.7 \\
\hline Ciudad Juárez & 69 & 36 & 2.6 & 2.3 & 2.3 & 3 & 2.7 & 2.3 & 2.1 & 2.7 \\
\hline Reynosa & 68.3 & 39 & 2.7 & 2.5 & 2.4 & 2.9 & 2.9 & 2.2 & 2.1 & 2.6 \\
\hline Tijuana & 66.7 & 46 & 2.6 & 2.3 & 2.1 & 3 & 2.6 & 2.5 & 2.2 & 2.7 \\
\hline
\end{tabular}

Fuente: Elaboración propia con base a Gabinete de Comunicación Estratégica Gce (2014). i. Movilidad, ii. Medio ambiente, iii. Oferta de vivienda, iv. Centros de diversión, v. Escuelas, vi. Museos y espacios históricos, vii. Naturaleza, viii. Ambiente de convivencia.

Las variables mejor evaluadas por los ciudadanos de Hermosillo son Centros de diversión y Escuelas, las que obtuvieron menor percepción son Museos y espacios históricos, y Naturaleza. Los juarenses determinaron a los Centros de diversión, las Escuelas y al Ambiente de convivencia como las mejores variables, mientras que Medio ambiente, Oferta de vivienda y Museos y espacios históricos (CUADRO 4).

Complementando a los resultados generados, la trascendencia de evaluar y medir diversos aspectos gubernamentales desde la sociedad civil organizada, reside en la percepción social de que en el país en general y en los estados de Chihuahua y Sonora en particular la corrupción se ha vuelto un problema importante que requiere urgente atención. La segunda Encuesta Nacional de Calidad e Impacto Gubernamental (INEGI, 2014) señala a la corrupción como el tercer problema más importante tanto a nivel nacional como para el estado de Chihuahua, en Sonora escala al segundo lugar (CUADRO 5), alarmante si se considera que acapara las mismas preocupaciones que otros problemas de tipo estructural 
como el desempleo y la inseguridad y la violencia, y se encuentra por arriba de necesidades básicas como vivienda, salud, alimentación.

Cuadro 5 - Percepción ciudadana (nacional y estatal) de los problemas más importantes

\begin{tabular}{|l|l|l|l|l|l|}
\hline \multicolumn{2}{|c|}{ Chihuahua } & \multicolumn{2}{c|}{ Sonora } & \multicolumn{2}{c|}{ Nacional } \\
\hline $\begin{array}{l}\text { Inseguridad y } \\
\text { delincuencia }\end{array}$ & $76.7 \%$ & $\begin{array}{l}\text { Inseguridad y } \\
\text { delincuencia }\end{array}$ & $74.6 \%$ & $\begin{array}{l}\text { Inseguridad y } \\
\text { delincuencia }\end{array}$ & $70.4 \%$ \\
\hline Desempleo & $59.3 \%$ & Corrupción & $53.6 \%$ & Desempleo & $51.0 \%$ \\
\hline Corrupción & $48.0 \%$ & Desempleo & $48.1 \%$ & Corrupción & $48.5 \%$ \\
\hline
\end{tabular}

Fuente: Elaboración propia con base a INEGI (2014).

Aún más, las acciones sociales dirigidas a evaluar gobiernos son valiosas para generar credibilidad, certeza y confianza social entre un escenario de descrédito de la política ante los escándalos históricos presenciados por el pueblo mexicano, reflejados en la percepción sobre la frecuencia de corrupción: El $87.4 \%$ de los chihuahuenses y el $86.5 \%$ de los sonorenses afirman que la corrupción es "muy frecuente" y "frecuente"; ${ }^{10}$ se calcula que los actos de corrupción por cada 100,000 habitantes alcanzan los 44,006 en Chihuahua y los 9,458 en Sonora. ${ }^{11}$ Sobre el gobierno municipal, el $71.4 \%$ de los chihuahuenses y el $71.9 \%$ de los sonorenses perciben que la corrupción en ese nivel de gobierno es "muy frecuente" y "frecuente"12 (INEGI, 2014). Estas cifras son alarmantes ya para el Municipio de Hermosillo como para el de Juárez, si consideramos que el primero contiene al 29.46\% de la población total en el estado de Sonora y el segundo al $39.11 \%$ de la población chihuahuense.

\section{La acreditación ciudadana de la función contralora en los Municipios de Hermosillo, Sonora y de Juárez, Chihuahua}

\subsection{Elementos metodológicos}

La función contralora en el municipio mexicano se ha caracterizado, desde los orígenes de acciones de control gubernamental mediante la creación en 1971 de la Comisión de Coordinación y control del gasto público (LANZ CÁRDENAS; CÁRDENAS LANZ, 1993 , p. $402-403)^{13}$, pasando por leyes y reglamentos federales, estatales y reglamentos municipales, por padecer de falencias de diseño institucional, hechas para hacer parecer

$1088.3 \%$ promedio nacional.

1124,724 promedio nacional.

$1275.8 \%$ promedio nacional.

13 Aunque desde 1947 se expidió la primera Ley para el Control de los Organismos Descentralizados y Empresas de Participación Estatal, en 1966 la segunda Ley para el control (también paraestatal) y la tercera de 1970 (nunca reglamentadas) 
que el poder controla al poder, simulando en los últimos años la participación ciudadana mediante "contralorías ciudadanas" y acciones relacionadas a ese tipo.

El problema de investigación parte de un contexto en el cual a pesar de la ya larga existencia de contralorías municipales, persisten e incluso cada vez con mayor frecuencia acciones de corrupción, para lo cual se inició desde el gobierno federal, a través de reformas a la constitución el mes de mayo de $2015^{14}$, con la creación del Sistema Nacional Anticorrupción (SNA).

El SNA, que inició y se ha mantenido sin Fiscal Anticorrupción al menos hasta julio de 2017, previene la participación de actores sociales ${ }^{15}$ para la integración del SNA en sus Comités Coordinador (1), el de selección (5), y el de participación ciudadana (5), último que tiene entre muchas otras, la atribución siguiente:

Opinar o proponer, a través de su participación en la Comisión Ejecutiva, indicadores y metodologías para la medición y seguimiento del fenómeno de la corrupción, así como para la evaluación del cumplimiento de los objetivos y metas de la política nacional, las políticas integrales y los programas y acciones que implementen las autoridades que conforman el Sistema Nacional (LEY, 2016, p. 21-X).

Sin embargo y a pesar de reformas a la constitución y emisión de leyes secundarias, el Artículo 74 constitucional (Fracción VI), persiste en no sancionar el incumplimiento de objetivos de programas ${ }^{16}$, lo cual para ciudadanos interesados en la efectividad de la gestión municipal esto es demasiado relevante.

Por lo anterior, este reporte de investigación se avoca a atender esos vacíos: a construir y ofrecer en su caso a ese Comité de participación ciudadana un instrumento metodológico matricial de indicadores útil para medir efectos de satisfacción ciudadana de la función contralora sobre programas municipales, facilitando que la ciudadanía controle al poder.

Esa matriz de indicadores se ha diseñado (reconociendo méritos), a la luz e inspiración de la matriz de indicadores para la Gestión por Resultados para el Desarrollo (GpRD) que maneja el Inter-American Institute for Economic and Social Development (INDES) del Banco Interamericno para el Desarrollo (BID), con su metodología de diagnóstico SEPSN-Prodev, metodología ya ampliamente probada a nivel de gobiernos sub nacionales en diversos países de América. Ciertamente con variaciones significativas, se acoge ya no a los

14 Reforma a 14 artículos Constitucionales (22, 28, 41, 73, 74, 76, 79, 104, 108, 109, 113, 114, 116 y 122), promulgada el 27 de mayo de 2015.

15 El lector podría acudir para verificar al respecto a: http://www.gob.mx/sfp/acciones-y-programas/sistemanacional-anticorrupcion-64289, así como a la misma Ley general del Sistema Nacional Anticorrupción (LEY,2016), en sus Artículos 8 al 23.

16 El Artículo 74 constitucional sólo señala que en caso de incumplimiento de objetivos de programas la Auditoría Superior de la Federación (y demás organismos de control hasta el nivel municipal) sólo podrán emitir las recomendaciones para la mejora en el desempeño de los mismos, en los términos de la Ley 
cinco pilares de la $\mathrm{GpRD}^{17}$, sino atendiendo las cinco áreas que reglamentariamente operan en el Estado de Sonora para la función contralora del gobierno estata ${ }^{18}$, esto por razones obvias de que lo que se ha analizado ha sido la función contralora, y no a un gobierno sub nacional completo.

Los métodos de búsqueda de datos han sido necesariamente internos secuenciales, ya que en cada una de las contralorías municipales que han sido estudiadas se acudió a sensibilizar primero a actores clave de procesos de control interno a través de pláticas introductorias al proyecto de investigación, para entrevistarles posteriormente; esos "actores clave" se seleccionaron en términos de funcionarios del Ayuntamiento con atribuciones de control sobre el desempeño funcional, programático y/o presupuestal de las dependencias de la administración municipal. Así, internamente dentro de cada Ayuntamiento y de manera secuencial, uno tras otro, partiendo necesariamente de la cúspide organizacional a fin de lograr permisos de acceso, se fue obteniendo información relevante que incluso ellos mismos iban señalando como pertinente para la mejora de la función contralora desde intereses ciudadanos.

La técnica de evaluación ciudadana seleccionada es la "acreditación institucional", de la que deviene la herramienta de control construida ad hoc "Matriz de diagnóstico de la acreditación ciudadana de la función contralora". Tanto la técnica como la herramienta buscan la gestión evaluadora ciudadana de sistemas administrativos públicos con la finalidad de acotar la discrecionalidad gubernamental, fortaleciendo el diseño y la competitividad institucional.

Se buscó entonces generar una matriz de diagnóstico con indicadores agrupados por áreas: i. Control administrativo; ii. Desarrollo administrativo; iii. Control gubernamental; iv. Participación social y vinculación; y, v. Transparencia y combate a la corrupción. A cada área le corresponden 5 indicadores cuyas notas varían entre 0 y 1 punto de acuerdo al criterio de evaluación que se muestra en el cuadro 5.

El valor de cada área general se manifiesta así:

ag $=i_{1}+i_{2}+i_{3}+i_{4}+i_{5}$

donde:

ag = área general,

$\mathrm{i}=$ indicador.

17 Los cinco pilares de la GpRD son: Planeación, Presupuesto, Gestión financiera, Programas y proyectos, así como Seguimiento y evaluación por resultados.

18 Esas 5 áreas, señaladas en el Artículo 26 de la Ley orgánica del Poder Ejecutivo del Estado de Sonora (LEY, 2016-bis), son: Control y desarrollo administrativo; Desarrollo administrativo; control gubernamental; Participación social y vinculación, y finalmente el área de Transparencia y combate a la corrupción. 
Mientras que la del resultado de la acreditación final se manifiesta de la siguiente manera:

$\mathrm{af}=\mathrm{ag}_{1}+\mathrm{ag}_{2}+\mathrm{ag}_{3}+\mathrm{ag}_{4}+\mathrm{ag}_{5} / 5$

donde:

af $=$ acreditación final,

ag= área general.

Cuadro 5 - Criterios para evaluar por condición relativa

\begin{tabular}{|l|l|l|l|l|}
\hline \multicolumn{1}{|c|}{0 puntos. } & \multicolumn{1}{c|}{0.25 puntos. } & \multicolumn{1}{c|}{0.50 puntos. } & \multicolumn{1}{c|}{0.75 puntos. } & \multicolumn{1}{c|}{1 punto. } \\
\hline $\begin{array}{l}\text { No existe } \\
\text { evidencia } \\
\text { alguna de que } \\
\text { se registre o } \\
\text { cumpla con la } \\
\text { condición. }\end{array}$ & $\begin{array}{l}\text { Sólo existe } \\
\text { evidencia } \\
\text { de que la } \\
\text { condición inició, } \\
\text { pero no opera } \\
\text { documentada, } \\
\text { actualizada, o } \\
\text { protocolizada. }\end{array}$ & $\begin{array}{l}\text { La condición } \\
\text { inició, existen } \\
\text { elementos que } \\
\text { obligan, pero no } \\
\text { un sistema efectivo } \\
\text { documentado, } \\
\text { actualizado, } \\
\text { protocolizado. }\end{array}$ & $\begin{array}{l}\text { La condición } \\
\text { inició, existen } \\
\text { elementos que } \\
\text { obligan, pero la } \\
\text { efectividad de un } \\
\text { sistema documentado, } \\
\text { actualizado o } \\
\text { protocolizado no esta } \\
\text { probada. }\end{array}$ & $\begin{array}{l}\text { La condición } \\
\text { opera } \\
\text { documentada, } \\
\text { actualizada, } \\
\text { protocolizada y } \\
\text { con efectividad. }\end{array}$ \\
\hline
\end{tabular}

Fuente: Elaboración propia.

Nota 1- Que una condición se encuentre "actualizada" significa que existe un sistema de operación codificado y probado a cabalidad, puede ser un sistema, una norma, manual o programa, que genera certidumbre a las actividades gubernamentales revisadas y autorizadas de manera oficial con un máximo de tres ańos de antigüedad. Nota 2- Que una condición se encuentre "documentada" significa que existe un sistema de operación codificado y probado a cabalidad, puede ser un sistema, una norma, manual o programa que genera certidumbre a los procesos gubernamentales, aunque pudiese no estar actualizada.

Nota 3- Que un requisito se encuentre "protocolizado" implica la documentación de un proceso formalizado como norma, programa o manual.

Al cumplimiento de las áreas en términos de la nota final le corresponde una acreditación:

Acreditación primer nivel, nota de área y final entre 0 y 1.75 pts.

Acreditación segundo nivel, nota área y final entre 2 y 2.75 pts.

Acreditación tercer nivel, nota de área y final entre 3 y 3.75 pts.

Acreditación cuarto nivel, nota de área y final entre 4 y 4.75 pts.

Acreditación quinto nivel, nota de área y final de 5 pts.

Los resultados de este ejercicio de evaluación se presentan en el Cuadro 6. 
Cuadro 6 - Matriz de diagnóstico de la acreditación ciudadana de la función contralora. Municipio de Juárez, administración 2010 - 2013, Municipio de Hermosillo, administración $2012-2015$

\begin{tabular}{|c|c|c|c|c|c|c|}
\hline \multirow{2}{*}{ Indicador } & \multirow{2}{*}{ Descripción } & \multirow{2}{*}{\begin{tabular}{|c|} 
Valor: \\
Área/ \\
Indicador
\end{tabular}} & \multicolumn{2}{|c|}{$\begin{array}{c}\text { Nota: Área/ } \\
\text { Indicador }\end{array}$} & \multicolumn{2}{|c|}{$\begin{array}{c}\text { Nivel de } \\
\text { Acreditación }\end{array}$} \\
\hline & & & Jrz. & Hmo. & Jrz & Hmo. \\
\hline Área 1. & Control administrativo. & 5 & 4.50 & 3.75 & 4 & 3 \\
\hline 1.1. & Sistemas documentados de control. & 1 & 1 & 0.75 & & \\
\hline 1.2 . & Técnicas, instrumentos y procedimientos de control. & 1 & 1 & 0.50 & & \\
\hline 1.3 . & $\begin{array}{l}\text { Coordinación con dependencias de control de } \\
\text { poderes del estado, federación y municipios. }\end{array}$ & 1 & 0.50 & 0.75 & & \\
\hline 1.4 & $\begin{array}{l}\text { Compendio de bases normativas suficientes y } \\
\text { actualizadas, autonomía, sostenibilidad y resiliencia. }\end{array}$ & 1 & 1 & 1 & & \\
\hline 1.5. & $\begin{array}{l}\text { Políticas y estrategias para la implementación de la } \\
\text { Agenda de Buen Gobierno. }\end{array}$ & 1 & 1 & 0.75 & & \\
\hline Área 2. & Desarrollo administrativo. & 5 & 3.25 & 2.50 & 3 & 2 \\
\hline 2.1. & Comités de desarrollo institucional. & 1 & 0.75 & 0.50 & & \\
\hline 2.2 . & $\begin{array}{l}\text { Bienestar integral de empleados (física, anímica, } \\
\text { psicológica, espiritual y social-familiar). }\end{array}$ & 1 & 0.50 & 0 & & \\
\hline 2.3 . & Capacidad de creatividad e innovación institucional. & 1 & 0.25 & 0 & & \\
\hline 2.4 & $\begin{array}{l}\text { Simplificación administrativa, mejora regulatoria y } \\
\text { sistema de entrega recepción. }\end{array}$ & 1 & 1 & 1 & & \\
\hline 2.5 & $\begin{array}{l}\text { Imagen: Notas periodísticas, reconocimientos, } \\
\text { apertura. }\end{array}$ & 1 & 0.75 & 1 & & \\
\hline Área 3. & Control gubernamental. & 5 & 3.75 & 1.75 & 3 & 1 \\
\hline 3.1. & Sistema de medición y evaluación del desempeño. & 1 & 0.75 & 0.25 & & \\
\hline 3.2. & Sistema de control, auditoría y/o fiscalización. & 1 & 1 & 0.75 & & \\
\hline 3.3. & $\begin{array}{l}\text { Proceso de sanción de actos, omisiones o conductas } \\
\text { imputables a servidores públicos. }\end{array}$ & 1 & 1 & 0 & & \\
\hline 3.4 & $\begin{array}{l}\text { Sistema de congruencia programático presupuestal/ } \\
\text { normativa. }\end{array}$ & 1 & 0.75 & 0.50 & & \\
\hline 3.5. & Sistema de administración y control de riesgos. & 1 & 0.25 & 0.25 & & \\
\hline 4. & Participación social y vinculación. & 5 & 3.25 & 3.50 & 3 & 3 \\
\hline 4.1. & $\begin{array}{l}\text { Comités de contraloría social colaborando en } \\
\text { estrategias y mecanismos de vinculación ciudadana. }\end{array}$ & 1 & 0.50 & 0.50 & & \\
\hline 4.2 & $\begin{array}{l}\text { Sistema de atención a quejas, denuncias, peticiones, } \\
\text { sugerencias o reconocimientos. }\end{array}$ & 1 & 0.75 & 1 & & \\
\hline 4.3. & $\begin{array}{l}\text { Ciudadanos colaboran en la determinación de } \\
\text { metas, objetivos y estrategias de programas y } \\
\text { servicios. }\end{array}$ & 1 & 0.75 & 0.50 & & \\
\hline 4.4. & $\begin{array}{l}\text { Participan ciudadanos formados monitoreando y } \\
\text { aplicando diversas técnicas de control. }\end{array}$ & 1 & 1 & 1 & & \\
\hline 4.5. & $\begin{array}{l}\text { Concertación y aprovechamiento de capital social e } \\
\text { inteligencia colectiva. }\end{array}$ & 1 & 0.25 & 0.50 & & \\
\hline
\end{tabular}




\begin{tabular}{|c|c|c|c|c|c|c|}
\hline \multirow{2}{*}{ Indicador } & \multirow{2}{*}{ Descripción } & \multirow{2}{*}{\begin{tabular}{c|} 
Valor: \\
Área/ \\
Indicador
\end{tabular}} & \multicolumn{2}{|c|}{$\begin{array}{l}\text { Nota: Área/ } \\
\text { Indicador }\end{array}$} & \multicolumn{2}{|c|}{$\begin{array}{l}\text { Nivel de } \\
\text { Acreditación }\end{array}$} \\
\hline & & & Jrz. & Hmo. & Jrz & Hmo. \\
\hline Área 5. & Transparencia y combate a la corrupción. & 5 & 3.75 & 3.50 & 3 & 3 \\
\hline 5.1. & Transparencia suficiente para el ciudadano. & 1 & 1 & 1 & & \\
\hline 5.2. & $\begin{array}{l}\text { Índices y pruebas de transparencia y acceso a la } \\
\text { información. }\end{array}$ & 1 & 1 & 0.50 & & \\
\hline 5.3. & $\begin{array}{l}\text { Declaraciones patrimoniales anuales públicas } \\
\text { (proactivas) y precisas de directivos. }\end{array}$ & 1 & 1 & 1 & & \\
\hline 5.4 & $\begin{array}{l}\text { Apego a la Carta Iberoamericana de la función } \\
\text { pública. }\end{array}$ & 1 & 0.25 & 0 & & \\
\hline 5.5. & Apego al Código Iberoamericano de buen gobierno. & 1 & 0.50 & 1 & & \\
\hline \multicolumn{2}{|c|}{ Acreditación final } & & 3.75 & 3.00 & & \\
\hline \multicolumn{2}{|c|}{ Nivel de acreditación final } & & & & 3 & 3 \\
\hline
\end{tabular}

Fuente: Elaboración propia.

Un acercamiento general basado en la estrategia de solicitud y recolección de información así como en la interacción con los funcionarios públicos responsables de la misma, permite catalogar a este ejercicio útil para la consolidación del Gobierno Abierto en sus matices de colaboración con la sociedad y en transparentar información producto de los procesos internos, pero insuficiente para generar alternativas de solución a problemas sociales y en implicar de forma activa y real a los ciudadanos en la formulación y ejecución de políticas. La causa es la naturaleza de origen de este ejercicio: escudriñar procedimientos al interior de las administraciones municipales con base a la colaboración socio-gubernamental.

De un análisis general producto de los resultados arrojados por la matriz podemos afirmar que la administración municipal juarense 2010 - 2013, y la hermosillense 2012 - 2015 apuestan por la confianza y credibilidad ciudadana en la función pública a partir de ejercer sistemas documentados, actualizados, protocolizados y funcionales válidos para el control y desarrollo administrativo y gubernamental. Si bien es cierto que en el gobierno local hermosillense la maduración de gestión no se concretado, a diferencia del municipio juarense que ha favorecido en mayor grado al fortalecimiento de las capacidades institucionales, imprescindibles para legitimar las acciones del gobierno local (áreas 1, 2 y 3). En cuanto a la generación de capacidades organizativas orientadas al perfeccionamiento del funcionamiento interno, subrayando la trascendencia del capital humano, se determinó que el desempeño efectivo, la capacidad creativa y de innovación del personal no se aprovecha ni estimula. Es evidente la inexistencia de medidas y valoraciones adecuadas para la mejora sustantiva de la efectividad laboral a partir de la satisfacción de la plantilla laboral, sobre todo en Hermosillo, el gobierno de Juárez demostró mayor estructuración.

Por su parte, en ambos municipios cobran relevancia los resultados para impulsar la satisfacción, confianza y credibilidad ciudadana por medio de la medición objetiva y la asequibilidad de la información pública, así como la coordinación con organizaciones ciudadanas expertas para mejorar las consecuencias sobre materia de transparencia (áreas 4 y 5). Se valora también la normatividad que impacta a la apertura proactiva de servidores 
públicos por incorporarse a la rendición de cuentas sea en el ámbito personal o institucional. En estas áreas los resultados de ambas administraciones son muy similares. Se detectaron acciones válidas para transitar de un control gubernamental cerrado a la valoración desde el interés ciudadano sobre la rendición de cuentas como una acción de apertura de "gobiernos abiertos".

En este tenor sobresalen las acciones de ambos gobiernos por incorporar indicadores para el diagnóstico ciudadano, incluyendo el sistema de medición y evaluación del desempeño desde la satisfacción del usuario, con parámetros máximos y mínimos de cumplimiento de responsabilidades individuales e institucionales al lado de programas estímulo-sanción. Ambos mantienen pendientes en cuanto a la congruencia programática - presupuestal, ya que la correspondencia entre diversos instrumentos de gestión enfocados a metas objetivos por servicio o sector y los programas y políticas públicas, impera la necesidad de incorporar intereses y expectativas ciudadanas como el ejercicio de presupuesto colaborativo.

\subsection{El análisis de la información por área}

\subsubsection{Control administrativo}

El seguimiento al marco normativo que puedan afectar la operación del gobierno municipal está documentado, en algunos casos actualizados, el acceso a estos documentos es público a través de la página electrónica de transparencia. El reto para la administración de Hermosillo es garantizar la eficiencia (niel de acreditación 3), mientras que en Juárez es mejorarla (nivel de acreditación 4).

\subsubsection{Desarrollo administrativo}

Ambos gobiernos cuentan con varios comités de desarrollo institucional como el Comité de Planeación para el Desarrollo Municipal, Comité Municipal para la Prevención Social de la Violencia, el Comité de Desarrollo Urbano, a pesar de, la consolidación y eficiencia no es homogénea. Sobre el bienestar de los empleados, los retos son importantes, las mejoras se requieren desde los procesos de selección hasta el buen clima organizacional. No se detectó un programa de comunicación comunitaria que procure una retroalimentación efectiva entre los gobiernos y la sociedad útil, entre otras cosas, para conocer los impactos de las acciones municipales. La estrategia de mejora para Hermosillo debe perseguir la sistematización de las acciones aisladas (nivel de acreditación 2), mientras que el gobierno juarense deberá consolidar la sistematización (nivel de acreditación 3).

\subsubsection{Control gubernamental}

En ambos municipios trabajan dependencias para dar seguimiento al desempeño de las obras y acciones, a través de indicadores mensuales del gasto de inversión y de operación, así como del impacto social que tienen las actividades que cada una de las mismas realizan, sin embargo, entre ambos municipios hay grandes discrepancias, mientras que el juarense debe mejorar la sistematización para percibir el impacto social (nivel de acreditación 3), el de Hermosillo debe iniciar acciones para reunir evidencia (nivel de acreditación1). 


\subsubsection{Participación social y vinculación}

Se tienen identificados y documentados todos los comités de contraloría social ya que son requisito para operar programas federales, sin embargo, se detectó que no siempre su trabajo se vincula a la ejecución. El sistema de atención a quejas, denuncias, peticiones, sugerencias o reconocimientos está activo, pero insuficiente. Varias actividades municipales incentivan la participación ciudadana, están debidamente documentadas y se cuenta con un manual para este fin, pero no se cuenta con un plan para aprovechar el capital social y la inteligencia colectiva (Nivel de acreditación 3 para ambos municipios).

\subsubsection{Transparencia y combate a la corrupción}

Se cuenta con un portal de internet vinculado al Sistema INFOMEX $^{19}$ que recibe solicitudes de información por parte de los ciudadanos, mismas que son respondidas conforme a derecho, manteniendo la transparencia en asuntos públicos. Las administraciones municipales cuentas con módulos de información en cada una de las dependencias de gobierno cuya responsabilidad es mantener la Información Pública de Oficio en Carpetas a la vista de los ciudadanos y brindar la información que sea solicitada, tal como lo dicta la norma. Además de las evaluaciones hechas por organizaciones de la sociedad civil que se describen en el apartado 3 de este artículo, los gobiernos locales bajo la lupa se someten al escrutinio de instancias públicas estatales y nacionales como: la Auditoría Superior del Estado, la Secretaría de la Función Pública, la Secretaría de Hacienda y la Auditoría Superior del Gobierno Federal el reto es mejorar la eficiencia de estos esfuerzos (nivel de acreditación 3 para ambos municipios).

\subsection{Recomendaciones}

De los resultados obtenidos se permiten las siguientes recomendaciones.

i. Si bien los gobiernos locales trabajan con base a marcos normativos muy específicos, deben garantizar la calidad de los trabajos que se basan en la misma reglamentación.

ii. Se recomienda la detección y sistematización de buenas prácticas para mejorar los procesos burocráticos. No se debe admitir la falta de aprovechamiento del capital humano disponible.

iii. Implementar las actividades necesarias para incorporar a la ciudadanía a verdaderas prácticas de influencia, sobresale la importancia de la práctica de acreditaciones ciudadanas sobre funciones gubernamentales orientadas a su institucionalización, así como la de presupuestos participativos de dependencias municipales.

19 Es el sistema electrónico mediante el cual las personas presentan sus solicitudes de acceso a la información pública y de acceso, rectificación, cancelación y oposición de datos personales y es el sistema único para el registro y captura de todas las solicitudes recibidas por los sujetos obligados a través de los medios seńalados en la reglamentación vigente, así como para la recepción de los recursos de revisión interpuestos a través del propio sistema. 
iv. Asegurarse de que los diversos comités ciudadanos incluidos en algunas de las actividades de la administración pública logren su consolidación y se conviertan en verdaderos gestores del talento social.

v. Ambas administraciones municipales deben asegurar ambientes laborales propicios para la gestión del talento humano, apartando la cotidianidad y responsabilidad laboral de los intereses partidistas, así como proveer las condiciones suficientes para lograr el acceso igualitario y equitativo de todas las trabajadoras y todos los trabajadores a beneficios y mejoras laborales.

vi. Los gobiernos estatales deben legislar y los municipales reglamentar el apoyo financiero y dar apertura a Organizaciones de la Sociedad Civil para permitir a expertos en Gestión Socio-gubernamental actuar desde afuera de estructuras de gobierno permitiendo con ello a la vez, su gradual acreditación plena y apoyo en su caso para lograrla por cada función, dependencia y entidad de gobierno.

\section{Conclusiones}

Este ejercicio refleja indicios de madurez política ciudadana en el sentido de involucrarse en el funcionamiento interno de la administración municipal, por igual, este esfuerzo por inhibir la cultura de la opacidad muestra una relación horizontal sociedad gobierno, mediante la cual se colabora con diagnósticos sobre las tareas gubernamentales y sugiere alternativas de solución y mejora. Tanto la madurez política como la interacción horizontal, son dos condiciones sine qua non para alcanzar contextos suficientes de transparencia y la accountability.

La rendición de cuentas influye al gobierno abierto, aún más, es condición para su éxito, sin embargo, la acreditación de los informes relativos por parte de ciudadanos expertos organizados en redes es aún más legítimo y corresponsablemente efectivo, por lo cual es pertinente resaltar la importancia del trabajo en red entre una instancia gubernamental y grupos de ciudadanos. En los casos bajo comento, se concibió un trabajo intenso de colaboración a partir de la apertura gubernamental para considerar intereses ciudadanos, aunque habrá que subrayar áreas de mejora en rubros como la confianza y la calidad de la información proporcionada.

En cuanto a las condiciones para el gobierno abierto, la evaluación detectó prácticas relativas, como el desarrollo de un sistema de indicadores para evaluar la gestión, iniciativas de contraloría social y sobre todo, el acceso a la información pública mediante el uso de las tecnologías de la información y comunicación. Es necesario otro tipo de ejercicios que discutan sobre tres elementos fundamentales: a).- Impulso a la institucionalización de la acreditación ciudadana de funciones cruciales para el desarrollo de instituciones gubernamentales, b).Generar alternativas conjuntas de solución a problemas gubernamentales, y c).- Implicar de forma activa y real a los ciudadanos en la formulación y ejecución de políticas. Un pendiente en general es la falta de un modelo municipal único para determinar gobiernos abiertos en los cuales los ciudadanos más que participar, colaboren comprometida y corresponsablemente en resoluciones de interés mutuo. 
Institucionalizar la incorporación de los intereses generales en los quehaceres municipales por medio de herramientas democráticas innovadoras, como la colaboración ciudadana para la acreditación de instituciones y dependencias gubernamentales en sus funciones, así como entre otras ya muy conocidas funciones tales como el presupuesto participativo, es un pendiente para alcanzar a plenitud efectos ya sea de transparencia, accountablity y de gobierno abierto. Este ejercicio de función social contralora sirve como continuidad de mediciones y evaluaciones sociales por detectar gobiernos que tengan como fin último garantizar la calidad de vida merecidas por la ciudadanía, es este caso las de Hermosillo y Juárez.

\section{Referencias}

CALDERÓN, César. Por qué un gobierno abierto? En: NASER, ALEJANDRA. Gastón Concha y Diana Parra. El desafío hacia el gobierno abierto en la hora de la legitimidad. Santiago de Chile, Cepal. 2012. Disponible en: <http://www.cepal.org/ddpe/ publicaciones/xml/9/46119/W465.pdf>. Consultado el 28 de julio del 2015.

EMMERICH, Gustavo. Transparencia, rendición de cuentas, responsabilidad gubernamental y participación ciudadana. Polis: Investigación y Análisis Sociopolítico y Psicosocial, v. 2, n. 4, p. 67-90, 2004.

EPSTEIN, Richard A. Concealment, use and disclosure of information. New Zaeland Business Roundtable, New Zaeland, 1996. 34 p.

FERREIRO, Alejandro. Dinero, política y transparencia: el imperativo democrático de combatir la corrupción. Ponencia presentada en la Novena Conferencia Anti-corrupción, Durban, Sudáfrica. 1999. Disponible en: <http://9iacc.org/papers/day1/ws3/dnld/d1ws3_ aferreiro.pdf $>$. Consultado el 25 de julio del 2015.

GCE - Gabinete de Comunicación Estratégica. Las ciudades más habitables de México 2014. 2014. Disponible en: <http://www.evalua.df.gob.mx/docs/gral/taller2015/ M01CMH.pdf>. Conslutado el 13 de agosto del 2015.

RUBIA, Antonio Garrido; MARTÍNEZ, María Antonia; PARRA, José Francisco. Accountability, democracia y reforma política en México. Siglo Veintiuno, 2011.

HERNÁNDEZ BONIVENTO, José Hernández. Diseño institucional para el gobierno abierto municipal: propuesta de medición y análisis del caso chileno. Documentos y Aportes en Administración Pública y Gestion Estatal, n. 27, p. 101-128, 2016.

HERNÁNDEZ, Patricia. Tendencias y primeros resultados de países a la vanguardia. En: NASER, Alejandra, Gastón Concha y Diana Parra. El desafío hacia el gobierno abierto en la hora de la legitimidad. Santiago de Chile, Cepal.2012. Disponible en: <http://www. cepal.org/ddpe/publicaciones/xml/9/46119/W465.pdf>. Consultado el 29 de julio del 2015. 
HEVIA, Felipe J. Construcción de capacidades estatales y patrones de relación Gobiernociudadanos en México: un análisis del nivel subnacional. Revista del CLAD Reforma y Democracia, n. 62, p. 107-134, 2015.

HUNGTINGTON, Samuel. La tercera ola de la democracia, En: Diamond Larry y Plattner Marc. El resurgimiento global de la democracia. México, UnAM. I996.

IMCO - Instituto Mexicano para la Competitividad A.C. Índice de herramientas electrónicas de gobiernos locales. 2015. Disponible en: <http://imco.org.mx/indices/ documentos/2015_IHE_Reporte_completo.pdf>. Consultado el 11 de agosto del 2015.

IMCO - Instituto Mexicano para la Competitividad A.C. Índice de Competitividad Urbana 2014. ¿Quién manda aquí? La gobernanza de las ciudades y el territorio en México. Disponible en: <http://imco.org.mx/indices/documentos/2014_ICU_Libro_ La_gobernanza_de_las_ciudades_y_el_territorio_en_Mexico.pdf $>$. Consultado el 10 de agosto del 2015.

IMCO - Instituto Mexicano para la Competitividad A.C. Índice de información Municipal presupuestal 2014. 2014b. Disponible en: <http://imco.org.mx/indices/ documentos/IIPM/2014_IIPM_Presentacion.pdf>. Consultado el 11 de agosto del 2015.

INEGI - Instituto Nacional de Estadística, Geografía e Informática. Resultados de la segunda Encuesta Nacional de Calidad e Impacto Gubernamental. 2014. Disponible en: <http:/www.inegi.org.mx/inegi/contenidos/espanol/prensa/Boletines/Boletin/ Comunicados/Especiales/2014/junio/comunica3.pdf $>$. Consultado el 2 de septiembre del 2015.

INEGI - Instituto Nacional de Estadística, Geografía e Informática. Censo General de Población y Vivienda. 2010. Disponible en: <http://www.inegi.org.mx/est/contenidos/ proyectos/ccpv/cpv2010/>. Consultado el 21 de agosto del 2015.

ARTHUR, Jones Lewis; KOLSON, Mildred Fogenpane. Cumplimiento del contrato psicológico y su implicación en el rendimiento de los empleados: El caso de Asanko Mina de Oro, Ghana. Journal of Public Administration and Policy Research, v. 9, n. 2, p. 17-25, 2017.

LANZ CÁRDENAS, José Trinidad; CÁRDENAS, José Trinidad Lanz. La contraloría y el control interno en México: antecedentes históricos y legislativos. Editorial Fondo de Cultura Económica, 2da. ed., México, 708 p. 1993.

LEY. Ley general del Sistema nacional Anticorrupción, DOF 18 de julio de 2016, México.

LEY. Ley Orgánica del Poder Ejecutivo del Estado de Sonora, actualizada al mes de septiembre de 2016. 2016-bis 
LÓPEZ, Sergio. La creación de la Ley de Acceso a la Información en México: Una perspectiva desde el Ejecutivo Federal, en, Hugo Concha, Sergio López y Lucy Tacher (coordinadores), Transparentar al Estado: la experiencia mexicana de acceso a la información. Instituto de Investigaciones Jurídicas. México, UnAM. 2005. Disponible en: <http://www.bibliojuridica.org/libros/libro.htm?l=1407>. Consultado el 14 de agosto del 2015.

MAINWARING, Scott; BEJARANO, Ana Maria; LEÓN-GÓMEZ, Eduardo Pizarro. La crisis de la representación democrática en los países andinos. Editorial Norma, 2008.

MARINEEZ NAVARRO, Freddy. La dimensión relacional del gobierno abierto y el liderazgo colaborativo. Espiral (Guadalajara), v. 23, n. 65, p. 47-87, 2016.

MERINO, Mauricio (2005). Transparencia: libros, autores, ideas. México, Cide-IfaI. Disponible en: <http://inicio.ifai.org.mx/Publicaciones/tlai18.pdf>. Consultado el $11 \mathrm{de}$ julio del 2015.

MORALES MIGUEL, et al. Evolución de políticas gubernamentales desde la década de los años 70's en México, tendencia hacia la gestión socio-gubernamental para legitimar instituciones, en Memorias de la VI Conferencia anual de la Interamerican Network of Public Administration Education InPaE, Rosario, Argentina, mayo. 2014.

MORALES, Miguel; CASTRO, Ana Lilia Banda. Inclusión ciudadana en el diseño de estrategias de colaboración, en Memorias de la XI Conferencia Internacional InPAE, San Juan, Puerto Rico, mayo. 2015.

NASER, Alejandra; CONCHA, Gastón; PARRA, Diana. El desafío hacia el gobierno abierto en la hora de la legitimidad. Santiago de Chile, CePAL. 2012. Disponible en: <http://www.cepal.org/ddpe/publicaciones/xml/9/46119/W465.pdf>. Consultado el: 28 jul. 2015.

O’DONNELL, Guillermo. Democracia, Desarrollo Humano y Derechos Humanos. En: O’DONNELL, G.; IAZETA, O.; VARGAS C. J. Democracia, Desarrollo Humano y Ciudadanía). Santa Fe, Argentina, Homo Sapiens. 2003.

O’DONNELL, Guillermo. Accountability horizontal, en Estudios Políticos. México, UnAm, Núm. 19. 1998. Disponible en: <http://132.248.9.34/hevila/e-BIBLAT/CLASE/ cla149425.pdf>. Consultado el: 29 jul. 2015.

PETERS, Guy; MURILLO, Lorena. Gobernanza y burocracia pública: nuevas formas de democracia o nuevas formas de control? Foro internacional, v. 45, n. 4(184), p. 585-598, 2005 .

POLLO, Genc. Transparency and public participation: The Albanian case of open government partnership. Academicus: International Scientific Jorunal, n. 6, p 
35-40, 2012. Disponible en: <http://www.academicus.edu.al/nr6/AcademicusMMXII-6-035-040.pdf>. Consultado el: 27 ago. 2015.

PRUD'HOMME, Jean. Consulta popular y democracia directa. México, IfE. 1997. Disponible en: <http://www.ife.org.mx/documentos/DECEYEC/consulta_popular_y_ democracia_di.htm>. Consultado el: 20 jul. 2015.

REYES, Federico H. Corrupción: de los ángeles a los índices. México, IfAI. 2004.

TRANSPARENCY INTERNATIONAL. 2014 Corruption Perception Index. 2014. Disponible en: <http://www.transparency.org/cpi2014>. Consultado el: 29 de ago. 2015.

TRANSPARENCIA INTERNACIONAL. Índice de Percepción de la Corrupción, 2016. Disponible en: <http://www.tm.org.mx/wp-content/uploads/2017/01/mundial.png>. Consultado el: 19 jun. 2017

UVALLE, Ricardo. Gobernabilidad, transparencia y reconstrucción del Estado. Revista Mexicana de Ciencias Políticas y Sociales, v. L, n. 203, p. 97-116, 2008. Disponible en: <http://www.revistas.unam.mx/index.php/rmspys/article/view/41995/38119>. Consultado el 20 jul. 2014.

WEERAKKODY, Vishanth; REDDICK, Christopher. Public sector transformation through e-government. Experiences from Europe and North America. New York, Routledge. 2013. 